\title{
Platelet fibrinogen
}

\author{
P. A. CASTALDI ${ }^{1}$ AND J. CAEN ${ }^{2}$ \\ From the Department of Haemostasis, Institut de Recherches sur les Maladies du Sang, Hôpital \\ Saint-Louis, Paris, France
}

SYNOPSIS Platelet fibrinogen has been studied in normal, thrombasthenic, and hypofibrinogenaemic subjects. It has been differentiated into adsorbed (plasma) and extractable (intraplatelet) fractions. Isotopic studies suggest that exchange does not occur between intraplatelet and plasma fibrinogen and it appears possible that the intra-platelet fraction may be derived from the megakaryocyte. Six of nine thrombasthenic patients were found to have a severe deficiency of both adsorbed and extractable fibrinogen. Since the remaining three had near-normal platelet fibrinogen and all nine failed to aggregate it is improbable that the failure to adsorb fibrinogen is responsible for the defect in aggregation. Magnesium partially corrects adhesion to fibrin and clot retraction by these platelets, but has not been found to influence their fibrinogen adsorption. It is considered that the basic platelet surface defect, of varying severity, is responsible for the abnormalities of adsorption, aggregation, and adhesion in thrombasthenia. In the case of congenital hypofibrinogenaemia, fibrinogen transfusion corrects the long bleeding time, platelet-adsorbed fibrinogen, and the ability of platelets to spread on glass. It is possible that fibrinogen influences the surface properties of human platelets, although the final mechanism is not determined.

The role of fibrinogen in the earliest phases of haemostasis is, as yet, poorly elucidated even though it has been demonstrated that it is closely related to the platelet (Ware, Fahey, and Seegers, 1948; Seligmann, Goudemand, Janin, Bernard, and Grabar, 1957; Sokal, 1962), readily demonstrable in the tissues in dynamic exchange with the plasma (Gitlin and Borges, 1953), and, according to some authors (Duguid, 1959; Roos, 1957), adherent to vascular endothelium. Another argument for the involvement of fibrinogen in primary haemostasis rests on the fact that the bleeding time was reported longer than normal in congenital afibrinogenaemia (Alexander, Goldstein, Rich, Le Bolloc'h, Diamond, and Borges, 1954; Caen and Inceman, 1963).

Schmid, Jackson, and Conley (1962), working with trypsinized platelets, depleted of platelet fibrinogen, found no aggregation. In another paper (Jackson, Morse, Zieve, and Conley, 1963) they demonstrated that thrombasthenic platelets were deficient in a clottable protein. These two findings led the authors to suggest a relationship between platelet fibrinogen and aggregation, and this assumption was

'Supported in part by the Cooperation Technique, Paris, and by a travelling fellowship from the Craig Mostyn funds of the PostGraduate Medical Foundation, University of Sydney, Australia.

'Supported by EURATOM, contract No. 019-63-3-BIAF.

Received for publication 30 March 1965. discussed by Grette (1962) and Nachman (1964), both of whom found fibrinogen in extracts of trypsinized platelets. Nachman (1964) also found that another platelet protein is a substrate for the action of thrombin, supporting the previous immunological results of Salmon and Bounameaux (1958). The fact that afibrinogenaemic platelets aggregate normally under the influence of thrombin (Pinniger and Prunty, 1946; Caen and Inceman, 1963) is also strong evidence that fibrinogen is not the only platelet substrate in thrombin catalyzed aggregation, or that only minute quantities are required.

In an attempt to define more clearly the role of platelet fibrinogen, a study has been made of adsorbed and extractable fibrinogen in the platelets of normal, thrombasthenic, and hypofibrinogenaemic subjects. The results are discussed in the light of the existing haemostatic deficiencies. They do not support the hypothesis that platelet fibrinogen is related to the aggregation defect in thrombasthenia. It does appear possible that adsorbed platelet fibrinogen is involved in platelet spreading to glass and is related, in an undetermined manner, to the initial events of platelet adhesion in vivo.

\section{MATERIALS AND METHODS}

PREPaRATION OF PLATElets Blood was collected by clean venepuncture using siliconized glassware and 18 
gauge stainless steel needles. The anticoagulant was trisodium citrate $3.8 \%, 1$ part, to 9 parts of blood. All subsequent procedures were carried out at $4^{\circ} \mathrm{C}$. and nonwettable surfaces were employed up to the time of extraction of the platelets. The blood was centrifuged in glass tubes, each containing approximately $6.0 \mathrm{ml}$., for five minutes at $120 \times g$. The platelet-rich plasma was pooled and recentrifuged for two minutes at $380 \times g$ to remove any remaining red blood cells. The volume and platelet concentration were recorded and centrifugation repeated at approximately $6,000 \times g$ during 20 minutes. In this way virtually all platelets were harvested and the platelet sediments were free of red cell contamination.

WASHING AND EXTRACTION The platelets were washed by eight repetitions of suspension and sedimentation at $6,000 \times g$, in phosphate buffer $p H 7.9$ containing 0.0026 $\mathbf{M}$ ethylene diamine tetracetic acid, the volume of buffer being one half that of the original platelet-rich plasma. The final platelet button was homogenized by hand, using a teflon plunger and glass tube, in $1.5 \mathrm{ml}$. of the same buffer. Homogenization was continued until no intact platelets could be recognized with the phasecontrast microscope, the time required usually being approximately 30 minutes. The homogenate was removed, the tube washed with $0.5 \mathrm{ml}$. of buffer and washing and homogenate pooled and centrifuged for 30 minutes at $31,000 \times g$. The extract so obtained was saved and the sediment washed once by resuspension in $2.0 \mathrm{ml}$. of buffer and centrifugation at the same force for 20 minutes. The final sediment was homogenized to give a stable opaque suspension in $1.0 \mathrm{ml}$. of buffer. In some experiments the extraction procedure was repeated three or four times by homogenization of the sediment for 10 minutes and centrifugation. All wash fluids, a sample of the initial homogenate, the extract (s), and final homogenized sediment were tested immediately or otherwise stored at $-20^{\circ} \mathrm{C}$. fo one to seven days before testing.

A sixth part by volume of magnesium chloride $0 \cdot 10 \mathrm{M}$ was added to the citrated platelet-rich plasma of two of the thrombasthenic patients before washing and extraction.

Fibrinogen degradation products were prepared by six hours' incubation of human fibrinogen with an optimal concentration of streptokinase.

\section{TESTS FOR FIBRINOGEN}

AGGLUTINATION The antibody employed was supplied by Hyland Laboratories in a form bound to latex. ${ }^{3}$ Tests were performed on a mechanically rotated glass stage using direct light against a dark background. Equal volumes $(0.01 \mathrm{ml}$.) of undiluted test material and antibody suspension were mixed and the time of agglutination recorded.

PRECIPITATION Gel diffusion experiments were performed by the method of Elek-Ouchterlony 1948). Antifibrinogen antiserum was supplied by Hyland Laboratories.

In order to obtain detectable precipitation it was necessary to concentrate platelet extracts and washes

${ }^{3} \mathrm{Fi}$-Test, Hyland Laboratories, Los Angeles, California, U.S.A. approximately tenfold, by air drying at $4^{\circ} \mathrm{C}$. in dialysis sacs.

COAGULATION Samples of the platelet extracts ando washes, each of $0.2 \mathrm{ml}$, were mixed with $0.2 \mathrm{ml}$. of a흐 thrombin solution (Bovine Thrombin Parke Davis) con- $-\overline{\bar{D}}$ taining 50 units $/ \mathrm{ml}$. and incubated at $37^{\circ} \mathrm{C}$.

ISOTOPIC Iodine ${ }^{131}$-fibrinogen was supplied by Dr. P. ल) Amouch of the Centre National de Transfusion Sanguine, $\vec{\circ}$ Paris (Reuge, 1965). The preparation contained $1 \%$ protein which was $80 \%$ clottable. Two samples were used $\vec{\omega}$ with specific activities of 1 and $5 \mu \mathrm{c} / \mathrm{mg}$. fibrinogen respectively. A dose of $20 \mu \mathrm{C}$ was used in the transfusion? studies. A patient with a severe degree of congenital $\overrightarrow{ }$ hypofibrinogenaemia was injected on two occasions and a normal individual once. Both received oral iodine is before and during the experiments. Blood was collected before and at intervals after the injection and the isolated platelets treated as above. Undiluted platelet-free plasma, platelet wash fluids, and platelet fractions were counted $\vec{C}$ in a well-type sodium iodide scintillation counter.

\section{OTHER METHODS}

Bleeding time was determined by the Ivy technique. Platelet adhesion in vivo was assessed by the method of $\overrightarrow{0}$ Borchgrevink (1960). Platelet counts were made by theor method of Piette and Piette (1959). Spontaneous adhesion to glass and spontaneous platelet aggregation were assessed with the phase-contrast microscope. Plasma fibrinogen was measured by dry weight after coagulation with thrombin.

\section{THE PATIENTS}

Nine thrombasthenic patients were examined and the clinical and laboratory details will be the subject of another publication (Caen, Castaldi, Inceman, Larrieu, Leclerc, Probst, and Bernard, 1965). All satisfied the basic diagnostic criteria of normal platelet count, long:bleeding time, absence of platelet aggregation, and 3 . deficient or absent clot retraction.

Two patients with congenital hypofibrinogenaemia have been studied. The first has a plasma fibrinogen level of $15 \mathrm{mg}$. $/ 100 \mathrm{ml}$. and clinical details have been previously음 reported (Caen, Faur, Inceman, Chassigneux, Seligmann, $>$ Anagnostopoulos, and Bernard, 1964). At the time of study her peripheral platelet count was $120,000 / \mathrm{c} . \mathrm{mm} . \overline{\mathrm{N}}$ and the Ivy bleeding time longer than 15 minutes. Theo second has a plasma fibrinogen level of $1 \mathrm{mg} . / 100 \mathrm{ml} ., 0$ measurable only by immunological methods, and an prolonged Ivy bleeding time.

\section{RESULTS}

CONTROL STUDIES The latex-adsorbed antifibrino-gen utilized gave positive agglutination reactions ${ }^{-}$ with diluted plasma, diluted aluminium hydroxide- $\frac{}{\mathrm{D}}$ adsorbed plasma, normal serum, and fibrinogen $\frac{?}{9}$ degradation products to a protein concentration of 
$6.25 \gamma / \mathrm{ml}$. The reaction was negative with heated plasma $\left(56^{\circ} \mathrm{C}\right.$. for 30 minutes), bentonite-adsorbed plasma ( $15 \mathrm{mg} . / \mathrm{ml}$. plasma for 20 minutes), and the diluted plasma and undiluted and diluted serum of a patient with congenital afibrinogenaemia.

Prompt agglutination $(<15$ seconds) was observed with the fibrinogen preparation utilized at concentrations between 10 and $1,000 \gamma / \mathrm{ml}$. Below $10 \gamma / \mathrm{ml}$. agglutination was less intense and the end point more variable. An upper limit of 60 seconds was chosen to indicate a positive result. The results of the range of six such control experiments are presented in Table I.

\section{TABLE I}

AGGLUTINATION TIME WITH LATEX-ANTI-

FIBRINOGEN OF VARYING FIBRINOGEN CONCENTRATIONS

$\begin{array}{ll}\begin{array}{l}\text { Fibrinogen Concentration } \\ (\gamma / \mathrm{ml} .)\end{array} & \begin{array}{l}\text { Agglutination Time } \\ (\mathrm{sec} .)\end{array}\end{array}$

$\begin{array}{cc}10-1,000 & 6-15 \\ 2-5 & 11-56 \\ 2 & \text { Negative }\end{array}$

NORMAL PLATELETS Repeated tests were performed with control platelets, the final calculated platelet concentration in the extracted sediment ranging from 0.1 to $2.5 \times 10^{6} / \mathrm{c} . \mathrm{mm}$. In almost every case fibrinogen was detectable in all eight wash fluids and otherwise in the first five. The platelet extract was uniformly positive as was the initial homogenate and the homogenized sediment after the first extraction. Repetition of the procedure gave a positive result in the supernatant after a maximum of two further extractions. Thereafter extracts and homogenised sediment were negative. The initial sediment wash after the first extraction was uniformly negative. Thrombin, in the concentration employed, did not reveal clottable protein in any of the wash fluids or platelet extracts. These results are presented qualitatively in Table II, and Fig. 1 illustrates the results of gel-diffusion studies.

THROMBASTHENIC PLATELETS Two groups of results were found with the platelets of nine patients. In three cases there was detectable fibrinogen in all or nearly all wash fluids and in the platelet extract, although in the latter case agglutination times suggested concentrations less than the controls.

In the second group of six patients, traces of fibrinogen were detectable in a maximum of the first three wash fluids and usually confined to the first two. The homogenized platelet suspension was always strongly positive whereas the supernatant or first extract was always negative. A second extraction permitted the detection of this fibrinogen in the supernatant. Subsequent extracts were negative.
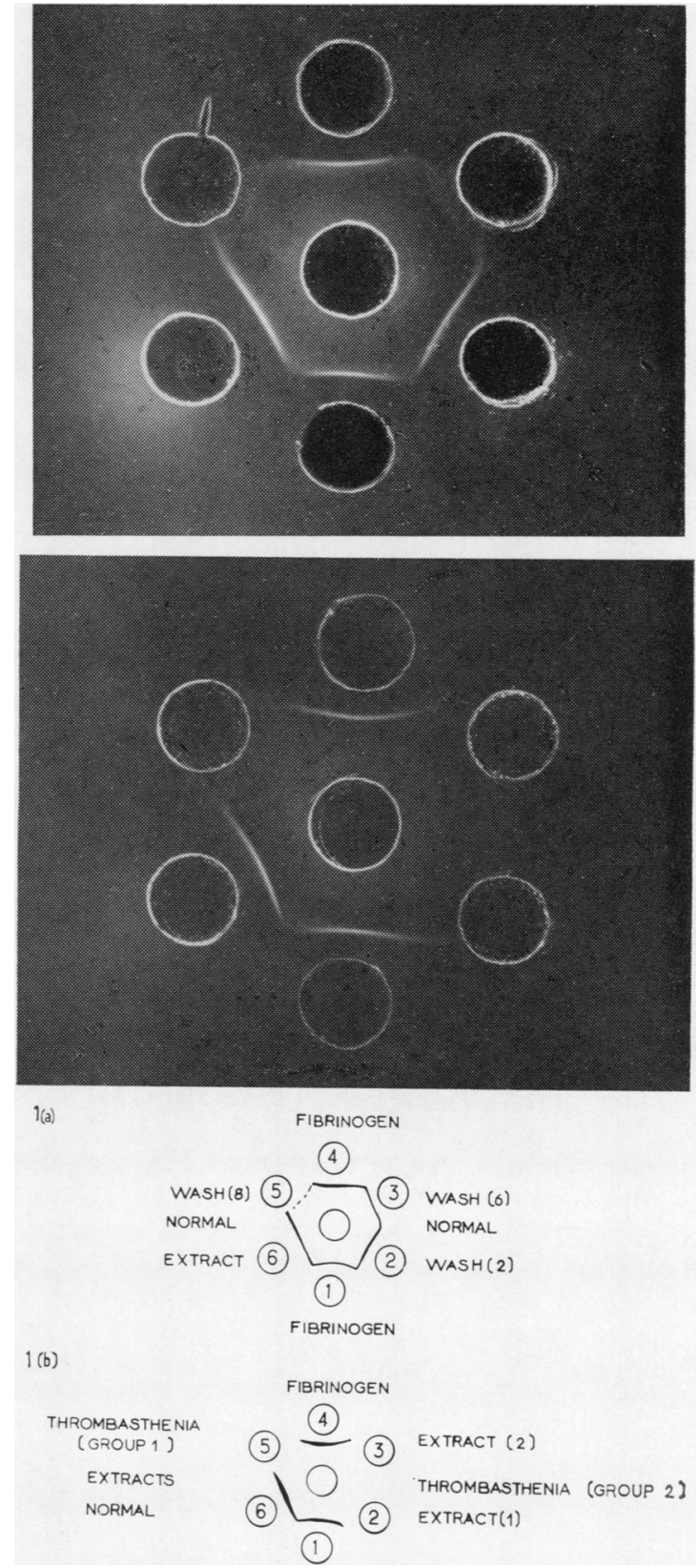

FIG. 1. Gel diffusion of concentrated platelet washes and extracts. The centre wells contained antihuman fibrinogen antiserum and wells number 1 and 4 a solution of purified human fibrinogen $(60 \mathrm{\gamma} / \mathrm{ml}$.). Wells 2,3 , and 5 (Fig. Ia) contained the second, sixth, and eighth washes of normal platelets and well 6 (Fig. Ia) a normal platelet extract. Wells 2 and 3 (Fig. 1b) contained successive extracts of the platelets of a thrombasthenic patient belonging to group 2. Well 5 contained the extract of a patient from group 1 and well 6 a different normal extract (Fig. Ib). Compare Table II. 
TABLE II

PLATELET FIBRINOGEN DETECTABLE BY IMMUNO-AGGLUTINATION ${ }^{1}$

Test

Normal

(6)

\begin{tabular}{ll}
\hline Group 1 & $\begin{array}{l}\text { Group } 2 \\
\text { (3) }\end{array}$
\end{tabular}

Wash fluid

2

3
4

5

8

Platelet homogenate

Extract 1

Sediment

Extract 2

Sediment

Platelet concentration

$\times 10^{6} / \mathrm{c} . \mathrm{mm}$.

${ }^{1}$ There is some correlation between agglutination time and fibrinogen concentration; +++ corresponds to a time less than $15 \mathrm{sec}$. and a concentration $10-1,000 \mathrm{\gamma} / \mathrm{ml}$.; ++ represents $16-30 \mathrm{sec}$. and $+31-60$ $\mathrm{sec}$. and correspond to concentrations less than $10 \mathrm{\gamma} / \mathrm{ml}$. The numbers of subjects tested are shown in parentheses. The platelets of one patient in group 2 thrombasthenia were tested twice and those of another in the same group, three times, with the same results.

These results are summarized in Table II and Figure 1. The addition of magnesium to the plateletrich plasma made no difference to the pattern of the results in the second group. There was considerable overlap between these two groups in the range of platelet concentrations tested (Table II).

hYPOFIBRINOGENAEMiC PLATELETS The platelet washes and extracts of the first patient (plasma fibrinogen $15 \mathrm{mg} . / 100 \mathrm{ml}$.) were tested on several occasions before and after transfusion of $4.4 \mathrm{~g}$. of fibrinogen. This transfusion was given at the same time as the second injection of labelled fibrinogen. Fibrinogen was detectable only in the first wash before transfusion, but three and 24 hours afterwards, when the plasma fibrinogen concentration was $62 \mathrm{mg}$. $/ 100 \mathrm{ml}$. and $52 \mathrm{mg}$. $/ 100 \mathrm{ml}$. respectively, a positive reaction was obtained with all eight washes. After $\mathbf{4 8}$ hours fibrinogen was detectable in the first two washes and thereafter in the first only. By the immunological method trace quantities of fibrinogen were detectable in the platelet extract both before and after transfusion (Table III). Figure 2 illustrates the coincident correction of the bleeding time and the disappearance curves of $\mathbf{I}^{131}$ fibrinogen in this patient and the control subject. After transfusion there were also observed an improvement in the adhesiveness in vivo index from $15 \%$ to $33 \%$ three hours after transfusion, and a
TABLE III

PLATELET FIBRINOGEN DETECTABLE BY IMMUNO-AGGLUTINATION ${ }^{1}$
Test

Normal
(6)
'Fibrinogen in washes and extracts of normal and hypofibrinogenaemic $\mathbb{D}$ platelets. Fibrinogen is absent from all but the first wash fluid before transfusion, but detectable in all wash fluids after transfusion. $\mathbb{D}$ Agglutination was positive before and after transfusion, in the platelet extracts of the patient.

significant degree of spontaneous spreading to glass and spontaneous aggregation, both absent before.

In the case of the second patient with extreme hypofibrinogenaemia (plasma fibrinogen $1 \mathrm{mg} . / 100$ ml.) platelet washes contained no detectable fibrinogen. However, trace amounts of this protein were found in the extract of those platelets.

ISOTOPIC STUDIES The half-time of disappearance of $I^{131}$ plasma fibrinogen was 66 and 68 hours in the $:$ two studies performed in the hypofibrinogenaemic 3 . subject and $\mathbf{7 4}$ hours in the control study (Figure 2.)

In the first experiment with the patient all platelet $₹$ washes and fractions were examined for radio- 0 activity. One hour after injection of the isotope, when plasma activity was maximal, there was significant radioactivity in the first five washes. Thereafter as plasma activity diminished, there was $O$ little detectable in any but the first wash fluid. At no $N$ stage throughout the entire period of study was there $\mathrm{W}_{\mathrm{J}}$ detectable activity in the platelet homogenate or 0 fractions (Table IV).

Following injection of the isotope in the control subject there was a small amount of radioactivity in $\stackrel{\mathcal{D}}{+}$ the eighth wash fluid of platelets collected 20 hours after injection. Fractions of platelets collected two $\overline{0}$ hours and 20 hours after injection did not contain $\mathbb{\mathbb { D }}$ any radioactivity. These results are summarized in $\bar{\alpha}$ Table IV. 


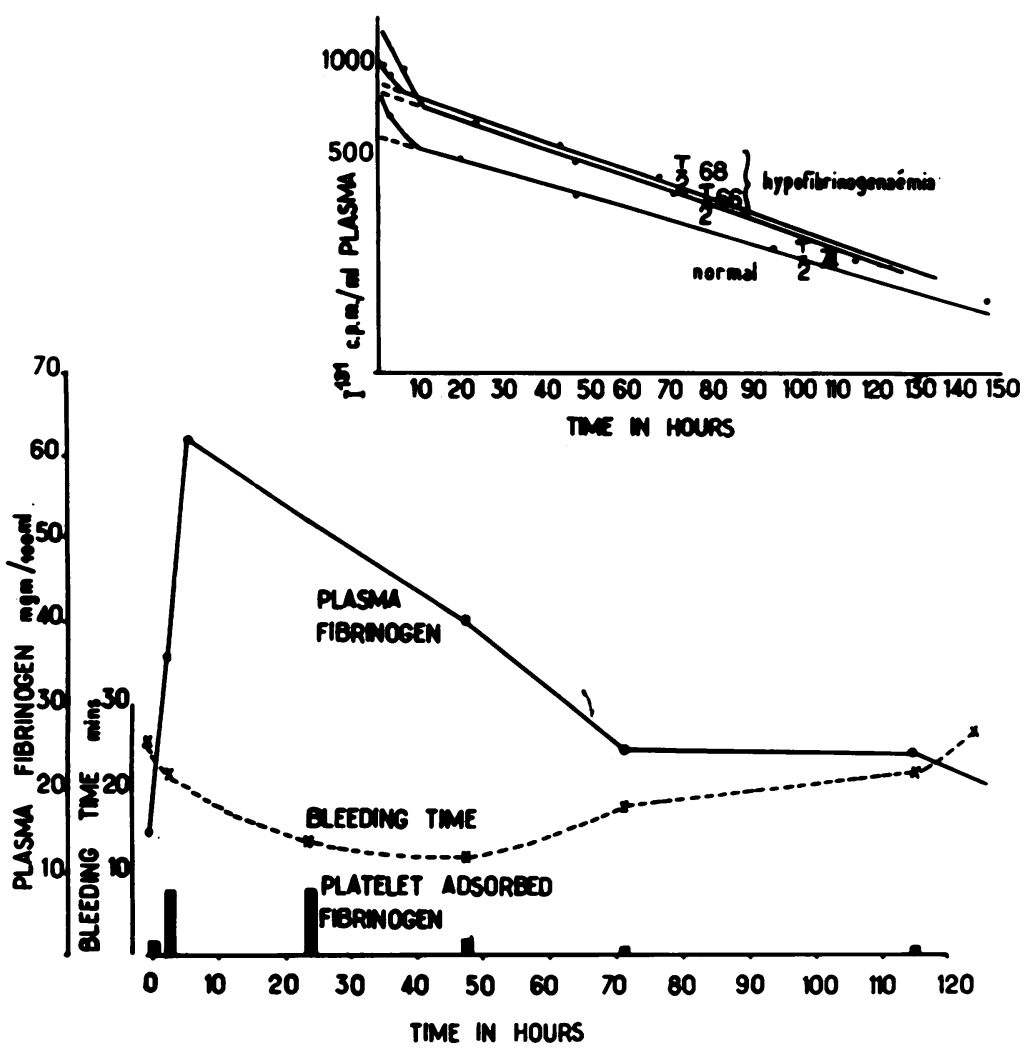

FIG. 2. The effect of transfusion of $4.4 \mathrm{~g}$. of fibrinogen in a patient with congenital hypofibrinogenaemia. Above are shown the disappearance curves of $I^{131}$-fibrinogen in the same patient and a control subject.
TABLE IV

RADIOACTIVITY OF PLATELET WASHES, PLATELETS, AND PLASMA AFTER INJECTION OF I $^{131}$-FIBRINOGEN

Test

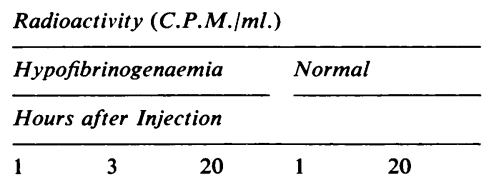

Wash number

$\begin{array}{lrrrrr}\text { Wash number } & 38 & 22 & 60 & - & 22 \\ 1 & - & - & - & - & - \\ 2 & 14 & 5 & - & - & - \\ 3 & 15 & - & 7 & - & - \\ 4 & 14 & 5 & 2 & - & 12 \\ 5 & 7 & 0 & - & - & 10 \\ 6 & - & - & 0 & - & - \\ 7 & 2 & 0 & - & - & 10 \\ 8 & 0 & 0 & 0 & 0 & 0 \\ \text { Platelets } & 956 & 880 & 458 & 775 & 448\end{array}$

${ }^{1}$ Radioactivity is detectable in platelet washes one hour (hypofibrinogenaemia) and 20 hours (normal) after injection of $20 \mu \mathrm{c}$. of $I^{131}$ fibrinogen. There is no radioactivity in the platelet fractions (indicates not tested).

\section{DISCUSSION}

Fibrinogen is clearly established as a platelet component although there has not been universal agreement as to its exact location. It is firmly adsorbed to the platelet surface and is not removed by multiple washing. Under the conditions of the present experiments it was not possible to demonstrate clottable protein in unconcentrated extracts, and the more sensitive immunological method was employed, expecially to demonstrate the nature of the quantitative abnormality in thrombasthenia. The findings of this study confirm and extend results previously reported (Castaldi, Caen, Seligmann, and Lasneret, 1964), when it was found that four of five thrombasthenic patients tested had deficient platelet fibrinogen. They also agree with similar observations from other laboratories (Jackson et al., 1963; Zucker, 1964).

The location and derivation of platelet fibrinogen now appear more clearly established. There seems little doubt that extractable fibrinogen is immunologically identical with plasma fibrinogen. The sole reservation in this regard must be the positive 
antibody reaction observed with its degradation products. Since it is so intimately adsorbed to the surface it is difficult to be sure that all detected in a platelet extract is entirely intracellular. The immunofluorescent findings of Sokal (1962) and Gokcen and Yunis (1963) indicated an intracellular component. The detection of extractable fibrinogen in the platelets of the hypofibrinogenaemic patients reported, when it was possible to be reasonably certain that all adsorbed fibrinogen was removed, is strong support for an intracellular fraction. In a recent study of subcellular platelet components, Nachman, Marcus, and Zucker-Franklin (1964) have demonstrated fibrinogen associated with the granules, which further supports such a location.

It was expected that the most likely source of intraplatelet fibrinogen would be the plasma protein pool. The isotopic findings reported did not support this possibility. It appears that, although the platelets of both the normal and hypofibrinogenaemic subjects adsorbed the labelled protein in the same manner as the non-radioactive fibrinogen, there was no detectable passage into the cell. It is therefore possible, as suggested by the results of Salmon and Bounameaux (1958), that intraplatelet fibrinogen is derived from the megakarocyte. Final confirmation of this suggestion would perhaps be found in the study of labelled precursors.

Some heterogeneity has been found amongst the thrombasthenic platelets examined. In the majority of cases there was little adsorbed platelet fibrinogen and gross deficiency of extractable fibrinogen. Three cases, however, had qualitatively normal fibrinogen although agglutination and diffusion studies suggested a slight quantitative decrease. Since aggregation is uniformly absent in all cases, whatever the stimulus, it appears unlikely that the inconstant deficiency of fibrinogen can account for this abnormality. It must therefore result from an underlying membrane disturbance, present in varying degrees of severity, always resulting in inaggregability, but sometimes permitting adsorption. The fact that fibrinogen was detectable, in subnormal amounts, in the extracts of these platelets when there was deficient adsorbed fibrinogen supports further the existence of an intraplatelet fraction.

The variability of the membrane defect is also indicated by differing degrees of fibrin adhesion observed in incubated and sectioned clots (Caen, Lasneret, and Michel, 1963). Clot retraction is also variably abnormal and may well be related to fibrin adhesion. In two of the three instances where the platelets demonstrate both adsorbed and extractable fibrinogen, previous studies (Castaldi et al., 1964) have indicated a degree of spontaneous fibrin adhesion and clot retraction. Furthermore the addition of mag- nesium has been shown (Caen et al., 1963) to induce. adhesion to fibrin and retraction, otherwise absent.. Magnesium was not found to increase fibrinogeno adsorption and hence its action would appear to beo independent of an increased affinity for fibrinogen. The mechanism of this effect remains unexplained.

The transfusion studies reported in severe hypofibrinogenaemia have shown a correction of the prolonged bleeding time and improvement in. platelet adhesiveness. Improvement in platelet $\overrightarrow{\vec{H}}$ spreading to glass after transfusion was previously observed by Gugler, Stillhart, Käser-Glanzmann, $\bar{\varnothing}$ and Lüscher (1962). Hence is appears that this property of platelets in vitro may have some relation 0 to haemostasis in vivo. Coincident with the increase or in plasma fibrinogen, correction of the bleeding 0 time and platelet spreading to glass there was 을 observed an increase in platelet surface fibrinogen by both immunological and tracer methods. It is there- $\omega$ fore possible that there may be a true correlation $\frac{\mathbb{D}}{0}$ between events in vitro and in vivo and the presence of $\frac{\mathbb{\Phi}}{3}$ fibrinogen on the platelet surface may contribute to the correction of haemostasis. Although some improvement in adhesiveness in vivo also occurred $\vec{\theta}$ we cannot be certain that adsorbed fibrinogen was of responsible and the sole factor in the correction of haemostasis.

In the absence of an effective concentration of plasma fibrinogen spontaneous spreading to glass and spontaneous aggregation are defective. In platelet-rich plasma of one of our patients (fibrinogen $15 \mathrm{mg}$./100 ml.) adenosine diphosphate (A.D.P.) and thrombin-induced aggregation were normal, whereas in the other case with a plasma fibrinogen of $1 \mathrm{mg}$./ $100 \mathrm{ml}$., A.D.P.-induced aggregation was delayed. It seems therefore that a minimal quantity of fibrinogen is required for platelet aggregation under these conditions. This assumption is supported by the improvement observed, after transfusion, in spontaneous spreading to glass and platelet cohesion, as assessed by phase microscopy. Fibrinogen may be essential to the initial contact reaction of platelets, and, in its absence, spreading may be defective or absent, A.D.P. release minimal or absent, and hence aggregation lacking.

Fibrinogen may be involved in the early phases of haemostasis through an effect on platelet adhesiveness. However it seems difficult to attribute the aggregation defect in thrombasthenia to a failure to adsorb fibrinogen and this more likely is the result of a basic surface disturbance. This surface abnormality would then be responsible for the failure to adsorb fibrinogen and perhaps other proteins, leading to defective fibrin adhesion and clot retraction and directly or indirectly to the failure of aggregation. 
Dr. Marjorie Zucker of New York, originally suggested the use of the latex-antifibrinogen reagent used in these studies and we express our gratitude to her for the suggestion and for sending to us the first batch of test reagent.

Human fibrinogen was supplied by Kabi, Stockholm, and fibrinogen degradation products by Dr. M. Seligmann and Dr. V. Marder. We are also indebted to Dr. Y. Najean who has done the isotopic measurements.

\section{REFERENCES}

Alexander, B., Goldstein, R., Rich, L., Lé Bolloc'h, A. G., Diamond L. K., and Borges, W. (1954). Congenital afibrinogenaemia (a study of some basic aspects of coagulation). Blood, 9, 843 .

Borchgrevink, C. F. (1960). A method for measuring platelet adhesiveness in vitro. Acta med. scand., 168, 157.

Caen, J., Castaldi, P., Inceman, S., Larrieu, M. J., Leclerc, J. C., Probst, M., and Bernard, J. (1965). Thrombasthenia. A review and report of fifteen cases. In preparation.

- , Faur, Y., Inceman, S., Chassigneux, J., Seligmann, M., Anagnostopoulos, T., and Bernard, J. (1964). Nécrose ischémique bilatérale dans un cas de grande hypofibrinogénémie congénitale. Nouv. Rev. franc. Hémat., 4, 321.

- and Inceman, S. (1963). Considérations sur l'allongement du temps de saignement dans l'afibrinogénémie congénitale. Ibid., 3, 614.

__ Lasneret, J., and Michel, H. (1963). Etude microscopique du caillot normal et pathologique (Les thrombasthénies constitutionelles et expérimentales). Effet du magnesium et de l'ATP. Ibid., 3, 251.

Castaldi, P., Caen, J., Seligmann, M., and Lasneret, J. (1964). Fibrinogen of thrombasthenic platelets. 10th Congr. int.Soc. Haematol., Stockholm. K.8.

Duguid, J. B. (1959). The role of the connective tissues in arterial diseases. In Connective Tissue, Thrombosis and Atherosclerosis, p. 13., edited by I. H. Page. Academic Press, New York and London.

Elek, F. D. (1948). Brit. Med. J., 1, 493.

Gitlin, D., and Borges, W. H. (1953). Studies on the metabolism of fibrinogen in two patients with congenital afibrinogenemia. Blood, 8, 679.
Gokcen, M., and Yunis, E. (1963). Fibrinogen as a part of platelet structure. Nature (Lond.), 200, 590.

Grette, K. (1962). Studies on the mechanism of thrombin-catalyzed hemostatic reactions in blood platelets. Acta Physiol. scand., suppl., 195.

Gugler, E. Stillhart, H., Käser-Glanzmann, R., and Lüscher, E. F. (1962). L'hémostase dans l'afibrinogénémie congénitale. Proc. Congr. int. Soc. Pediat., Lisbon.

Jackson, D. P., Morse, E. E., Zieve, P. D., and Conley, C. L. (1963) Thrombocytopathic purpura associated with defective clot retraction and absence of platelet fibrinogen. Blood, 22, 827.

Nachman, R. L. (1964). Immunologic studies of platelets protein. Personal communication.

- Marcus, A. J., and Zucker-Franklin, D. (1964). Subcellular localization of platelet fibrinogen. Blood, 24,853 .

Ouchterlony, O. (1948). Antigen-antibody reaction in gels. Ark. Kemi. Miner. Geol., 26, B(14).

Piette, M., and Piette, C. (1959). Numération des plaquettes sanguines utilisant un liquide hypotonique à base de chlorhydrate de procaine. Sang, 30, 144.

Pinniger, J. L., and Prunty, F. T. G. (1946). Some observations on the blood-clotting mechanism. The role of fibrinogen and platelets, with reference to a case of congenital afibrinogenaemia. Brit. J. exp. Path., 27, 200.

Reuge, C. (1965). Transfusion (Paris), in the press.

Roos, J. (1957). Blood coagulation as a continuous process. Thrombos. Diathes. haemorrh. (Stuttg.), 1, 471.

Salmon, J., and Bounameaux, Y. (1958). Etude des antigènes plaquettaires et en particulier, du fibrinogene. Ibid., 2, 93.

Schmid, H. J., Jackson, D. P., and Conley, C. L. (1962). Mechanism of action of thrombin on platelets. J. clin. Invest., 41, 543.

Seligmann, M. Goudemand, M., Janin, A., Bernard, J., and Grabar, P. (1957). Etudes immunochimiques sur la présence du fibrinogène dans des extraits de plaquettes humaines lavées et dans certains extraits leucocytaires. Rev. Hémat., 12, 302.

Sokal, G. (1962). Etude morphologique des plaquettes sanguines et de la métamorphose visqueuse au moyen d'anti-serums fluorescents antifibrinogène et antiplaquettes. Acta haemat. (Basel), 28, 313.

Ware, A., Fahey, J., and Seegers, W. H. (1948). Platelet extracts, fibrin formation and interaction of purified prothrombin and thromboplastin. Amer. J. Physiol., 154, 140.

Zucker, M. (1964). Platelet adhesion, release and aggregation. Thrombos. Diathes. haemorrh. (Stuttg.), suppl., 13, p. 301. 